\title{
ESREBP-— 一个检索和预报 稀土嗍酸盐绿色苂光粉的专家系统
}

\author{
安利群 李 正刘洪栗 陈念贻 \\ (中国科学院上海冶金研究所, 上海 200050) \\ 刘念红郭风瑜 \\ (北京大学化学系, 北京 100871)
}

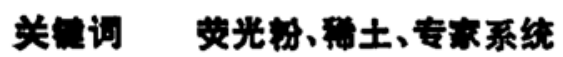

\section{一、引官}

专家系统研究是当今人工智能研究最活跃的领域之一. 从 60 年代中期开始, 专家系统在 许多领域都取得了成绩的, 而把专家系统技术用于稀土荧光粉的研制未见报道. 稀土三基色 荧光粉自 60 年代出现以来,国内外在其研制方面做过大量工作,已有实验结果表明,这类材料 成分复杂, 最佳工艺条件难以摸索, 是合成难度较大的新材料. 人工智能的另一领域一模式 识别与化学键参数法结合在新化合物预报, 地质勘探、工业优化 ${ }^{[2]}$ 等方面亦取得很大成功.

本文报道一个以 Turbo-Prolog 为核心语言的基于逻辑的专家系统. 该系统除了具有一 般专家系统所具有的特点外 (如灵活可变的知识库, 友善的人机交互系统等), 还运用模式识别 技术将专家们近十几年所做出的杂乱繁多的绿粉配方进行分类计算,找到了良好的规律性,实 现了绿粉配方的预报. 并以预报配方作实验, 合成出了亮度高于标准绿粉 $\mathrm{MgAl}_{11} \mathrm{O}_{19}: \mathrm{Ce}, \mathrm{Tb}$ 的新荧光粉,证明了系统的可靠性.

\section{二、系 统 功 能}

本专家系统具有如下几方面功能:

1. 查询检案功能 (1)根据苂光粉的发光性能, 检索配方; (2)根据配方, 检索工艺条件; (3)根据配方, 检索发光性能.

2. 顶报功能 (1)根据配方, 预报亮度; (2) 预报亮度大于 $180 \%$ （vs. (Ce, Tb) $\mathrm{O}_{3}$. $\left.3 \mathrm{~B}_{2} \mathrm{O}_{3}\right)$ 的配方, 配方中组分种类和含量可由用户适当选择, 该部分配备解释指导, 可引导用户 逐步完成模式识别预报工作.
3. 知识库的维护
(1)补充知识; (2)删除知识; (3)将修改后的知识库存盘.
4. 模式识别功能 可进行 PCA、NLM、ODV、ODP 等方法的分析 ${ }^{[3]}$.

\section{三、预 报 原 理}

预报是根据模式识别的分析结果进行的, 训练集来自文献 [4]中的 44 个配方, 以相对亮度 1991-01-28 收稿,1992-04-04 笅佟改稿 
大于 $180 \%$ 的配方为 1 类点, 小于 $180 \%$ 的配方为 2 类点. 相对标准是 $(\mathrm{Ce}, \mathrm{Tb})_{2} \mathrm{O}_{3} \cdot 3 \mathrm{~B}_{2} \mathrm{O}_{3}$, 取 6 个特征变量：(1) $\mathrm{Tb}$ 含量; (2) $\mathrm{Ce}$ 含量; (3) $\mathrm{B}_{2} \mathrm{O}_{3}$ 含量;(4) $\mathrm{MgO}$ 含量;(5)基质的平均 键参数 $Z / r(Z$ 为原子序数, $r$ 为离子半径, 对组分的摩尔分数取权重平均 $)$; (6)基质的平均 电负性(对组分的算术平均). 用模式识别中的 PCA 法计算分析后，分类成功. 如图 1 所示. 根据模式识别的原理, 如未知样本 (配方) 经 PCA 计算属高亮度区, 则该配方应具高亮度. 反 之, 从亮度高的区域出发, 由主成分空间返回原始空间可逆算出新配方, 这便是预报亮度和配 方的根据。

\section{四、系统 结 构}

系统主要由知识库、推理机、用户接口系统及外部程序等几大模块组成.

1. 知识库在系统中知识库主要由说明事实和数据的逻辑谓词子句组成, 这些子句合 成组成 Torbo-Prolog 数据库, 存在一磁盘文件中. 知识包括近年来国内外最新的荧光粉配 方, 相对亮度, 寿命、高温性能、工艺条件和化学键参数如电负性、离子半径、原子序数等. 知识 主要来自三方面: (1)专利;(2)文献手册;(3)实验数据.

2. 推理机 ESREBP 系统采用逆向链接推理策略, 这个策略和纠错规则一起, 用来分离 错误和产生咨询, 推理通过搜索和模式匹配完 成. 由 Turbo-Prolog 中的内部合一子程序自 动完成. 推理结束时, 顶层规则通过用户接口 将结果告诉用户。

3. 用户接口系统该部分主要由提供菜 单和清窗口的规则组成, 亦包括控制用户对程 序功能的选择和与用户的通信. 由内部谓词 readint, realreal 等接收用户问答,write 谓词 输出结果给用户。

\section{4. 外部程序及接口 由于模式识别预报} 需要大量计算, 而 Turbo-Prolog 等智能化语 言都缺乏过程式语言的计算能力, 此方面的不

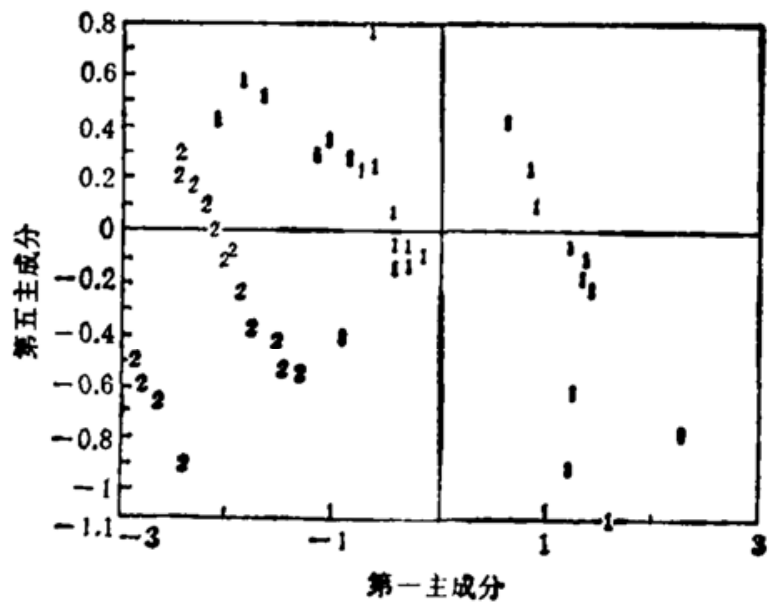

图 1 PCA 法分类图

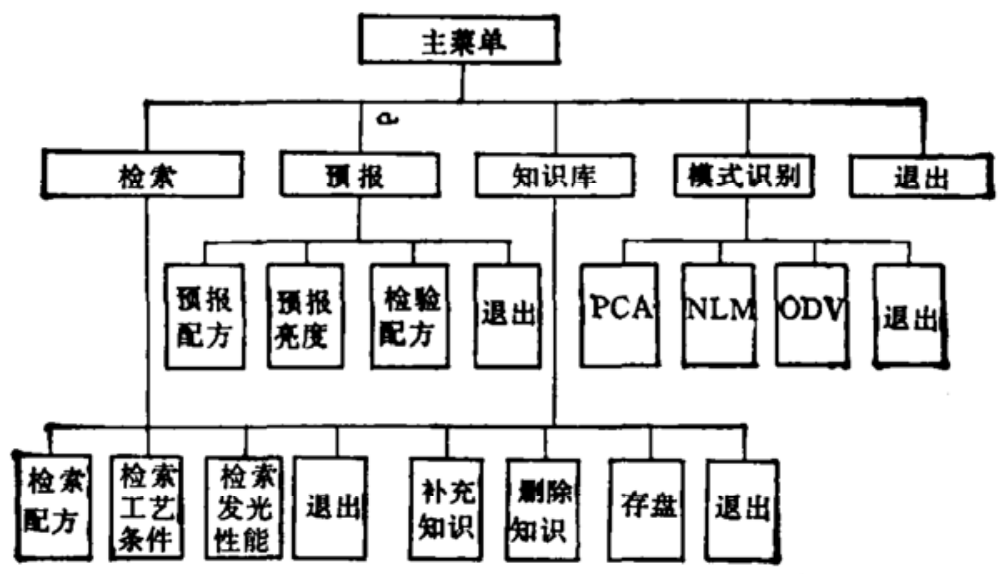

图 2 ESREBP 系统的层次图 
足可通过调用外部程序来弥补,涉及算法的模式识别程序是用 BASIC 语言编写的,编译成执 行文件后,通过 System (XXX·EXE) 命令调用此程序,运行完毕返回 Prolog 环境。

5. 目形承统主要用于模式识别预报。从图上用户可直观地看到预报配方是否合乎模 式识别预报标准,在临界状态可由用户决定取舍.系统层次图见图 2.

\section{五、系统应用兴例}

这里给出预报配方的部分示例. 在运行 ESREBP 系统后, 显示主菜单, 选择“预报”后,显 示预报分菜单,选择“预报配方”项，即给出预报的有关操作说明和模式识别分类图，以便用户 了解样本点的分布,之后屏幕显示:

Enter relative brightness (vs. $(\mathrm{Ce}, \mathrm{Tb})_{2} \mathrm{O}_{3} \cdot 3 \mathrm{~B}_{2} \mathrm{O}_{3}$ ): 2004

Do you want to set variables $(y / n)$ ? $\mathrm{Y} \leftarrow$

How many variables do you want to set? $1 \leftarrow$

which variable? ।

$$
\text { set value }-0.03
$$

上述显示指预报相对亮度约 $200 \%$ 的配方, 且设置变量 1 (即 $\mathrm{Tb}$ 含量)为 0.03 , 以后系统 将算出原始变量的值并进一步求算配方, 此时系统将询问您希望在配方中掺人何种元素:

Enter atomic number of element (1)-11(Na)

Enter atomic number of element (2)-20 (Ca)

Enter atomic number of element (3)-39(Y)

系统将调用 $\mathrm{Na} 、 \mathrm{Ca} 、 \mathrm{Y}$ 三元素的键参数求算它们在配方中的适宜含量,给出完整的配方 供用户参考。

利用本系统预报的配方, 我们合成出了若干成本较低、亮度高于标 准 绿 粉 (Ce, Tb) $\mathrm{Mg} \mathrm{Al}_{11} \mathrm{O}_{1}$ 的新嗍酸块绿粉.

\section{* 文 喽}

[1] 张吉策、承安、贾洁之, 专家系统与知识工程引论, 消华大学出版社, 北京, 1988 .

[ 2] 炼念贻等,计算化学及其应用,上海科技出版社, 1987 .

[ 3 ] 本介谷等, 计算机棋式识别技术, 上海交通大学出版社, 1986 .

[4] Kasei, Optonix Ltd, Tokio, Ger. Pat., 3029389.

[5]南京大学计策机系编, Turbo Prolog 程序设计, 南京大学出版社, 1988 . 